\title{
Negative genetic correlations between production traits and head or bony tissues in large all-female rainbow trout (Oncorhynchus mykiss)
}

\author{
Pierrick Haffray ${ }^{\mathrm{a}, *}$, Jérôme Bugeon ${ }^{\mathrm{b}}$, Cédric Pincent ${ }^{\mathrm{a}}$, Hervé Chapuis ${ }^{\mathrm{c}}$, Emmanuel Mazeiraud $^{\mathrm{d}}$, \\ Marie-Noelle Rossignol ${ }^{\mathrm{e}}$, Béatrice Chatain ${ }^{\mathrm{f}}$, Marc Vandeputte $^{\mathrm{f}, \mathrm{g}}$, Mathilde Dupont-Nivet ${ }^{\mathrm{g}}$
}

\author{
a SYSAAF, Station SCRIBE, Campus de Beaulieu, 35042 Rennes, France \\ b INRA, UR1037, SCRIBE, IFR 140 GFAS, Campus de Beaulieu, F-35042 Rennes, France \\ ${ }^{c}$ SYSAAF, SRA/INRA, F-37380 Nouzilly, France \\ d Aqualande, Route de St Gor, F-40120 Roquefort, France \\ e LABOGENA, Domaine de Vilvert, F-78350 Jouy-en-Josas, France \\ ${ }^{\mathrm{f}}$ Ifremer, UMR110 INTREPID, Chemin de Maguelone, F-34250 Palavas-les-Flots, France \\ ${ }^{9}$ INRA, UMR1313 Génétique Animale et Biologie Intégrative, F-78350 Jouy-en-Josas, France \\ *: Corresponding author : Pierrick Haffray, Tel.: + 33223485378 ; fax: + 33223485020 ; \\ email address : haffray@rennes.inra.fr
}

\begin{abstract}
:
Genetic parameters of production traits (growth, carcass yield, fillet yield) and bony tissues (head and vertebral axis) were estimated for large all-female rainbow trout Oncorhynchus mykiss reared in freshwater. Genetic parameters were estimated using REML at 16 months of age (1636 g) on 1962 DNA-assigned progenies from a partial factorial mating design with 60 dams and 100 sex-reversed sires.

Most traits presented medium to high heritability (0.37 to 0.54$)$. A high genetic correlation $\left(r_{A}=0.97\right)$ was found between fillet yield and headless gutted carcass yield (or HGCarc\%). Due to its higher heritability and high genetic correlation with fillet yield, selection on HGCarc\% should result in a $50 \%$ increase in selection efficiency on fillet yield by reducing operator-linked variability at filleting. However, strong negative genetic correlations were estimated between body weight or fillet yield and bony tissue development as head yield or the head and vertebral column yield $(-0.48$ to -0.57$)$. Ten generations of selection on body weight (or fillet yield) are, therefore, predicted to decrease the relative head development by 25 to $30 \%$ for slaughtering at constant age. As it is impossible to disentangle this result from a correlation that is only mathematically determined, this result needs further investigations. If yields are corrected according to their allometric relationship with body weight, only selection for fillet yield will decrease the proportion of bony tissues for a slaughtering at a constant body weight. Whatever the final objective of selection (to increase body weight or to shorten the production cycle at constant body weight), it is concluded that at least selection on fillet yield will affect the relative head and the vertebral axis development and that selection on growth may affect bony tissue development. These results are discussed in relation to the past results from livestock breeding, resource energy allocation theory and a future improvement of robustness by selection.
\end{abstract}

\section{Highlights}

Genetic parameters of production traits in rainbow trout - Negative correlation between production traits and bony tissues Selection on fillet yield

Keywords : Resource allocation ; Fillet yield ; Head ; Salmonids ; Aquaculture ; Selective breeding 


\section{Introduction}

Improvement of growth and meat yield (production traits) were among the first selection objectives in the history of livestock production. Dramatic improvements for these traits have been achieved by artificial selection in poultry and other livestock (Havenstein et al., 2003a, b; Mignon-Grasteau et al., 2005; Rauw, 2009). Improvement of the edible meat ratio can also be considered to improve the ecological efficiency of animal production, as more edible meat is produced using the same input levels (feed, labour and energy). On the other hand, significant undesirable side effects of selection for increased growth and meat yield have also been reported for non selected functional traits (Rauw et al., 1998; van der Most et al., 2011). The relative increase of the volume of one body component (such as the musculature) is logically associated with a decrease in the relative size of other body components. In poultry, broilers selected for growth and high breast yield have a smaller lung volume (- 20 to - $30 \%$ ) and an increased susceptibility to Sudden Death Syndrome, defined as pulmonary hypertension complicated by ascites (Sanchez et al., 2000; Siegle et al., 2009). The same kind of detrimental effects are also observed in pigs, where highly productive breeds have lower blood volumes and smaller cardiovascular systems (Knap and Rauw, 2009). In farmed fish, the genetic progress for growth achieved though commercial selection remains poorly documented. Experiments report +10 to $30 \%$ genetic gains per generation, which are similar to or higher than the gains achieved in poultry selection per generation (see the review by Gjedrem and Thodesen, 2005; Vandeputte et al., 2009a). Unexpectedly, the heritability of fillet yield in fish is highly variable, ranging from 0.03 to 0.38 (Kause et al., 2002; Neira et al., 2004; Rutten et al., 2005; Kause et al., 2007, 2011; Kocour et al., 2007; Powell et al., 2009; Saillant et al., 2009; Navarro et al., 2009; Nguyen et al., 2010; Kause et al., 2011; Gjerde et al., 2012) and exhibits very diverse genetic correlations with body weight (-0.02 to 0.98). If fish traits were to show the same response to selection as the other animal examples mentioned above, an increase in fillet yield would be expected to decrease some other body compartments in relative volume. The estimation of heritability of non-selected functional traits (such as bony tissues) and their genetic correlations with growth and processing traits (such as fillet yield) is, therefore, crucial for the design of sustainable breeding programs for aquaculture species (Komen et al., 2002).

The goal of this experiment was to estimate the genetic basis of relative head and bone development in rainbow trout and the interaction of these traits with the growth of other body compartments represented by commercial traits such as fillet yield and carcass yield. Results of this study should be of use to fish breeders to foresee potential undesirable side effects of selection for high productivity.

\section{Materials and methods}

\subsection{Experimental population}

The fish used were derived from a commercial line from the Aqualande breeding company (France), which had already been selected for growth for 3 generations using improved PROSPER principles (Chevassus et al., 2004) integrating also selection on external morphology and pedigree tracing with DNA parentage assignment (Haffray et al., 2004). The inbreeding ( $F$ ) level of the population estimated with the "pedigree" package of the $R$ software was $0.45 \%$. 
Six hundred all-female full-sib families were produced the same day at the Aqualande breeding centre (Pissos, France). Sixty dams (2 years of age) were crossed with 100 sexreversed females (defined as sires) in 10 full factorial crosses of 6 dams by 10 sires according Dupont-Nivet et al. (2006). Half-sib families from the different dams were incubated together so as to limit early non-genetic maternal effects. Further details can be found in Haffray et al. (2012). Briefly, each spawns were incubated separately. At eyed stage, 12 groups of 5 spawns each were created based on mean eyed egg size of each spawn to limit within-group mean egg weight differences between spawns. The initial mean weight differences between groups were progressively decreased by using different feeding ratios for each group as described and already discussed (Haffray et al., 2012). When groups achieved the same mean body length (147 days post fertilization (dpf); $9.7 \mathrm{~cm}$ ), 250 individuals per group were pooled (in total 3000 fish). This procedure was efficient to limit subsequent environmental tank effect and non-genetic maternal effect dropped to 0 for the traits considered (body weight, condition coefficient; Haffray et al., (2012)). At 198 dpf, fish were individually tagged with RFID transponders. Their DNA was collected through a fin sample preserved in $95 \%$ ethanol. At $220 \mathrm{dpf}$, fish were vaccinated against Yersinia ruckeri and transferred to the "Viviers de la Hountine" fish farm (Belin-Béliet, France) located $12 \mathrm{~km}$ downstream the Pissos hatchery.

In the two fish farms, rearing units were supplied with flow-through river water just entering in the fish farm. The water temperature varies from 3 to $20^{\circ} \mathrm{C}$ and oxygen concentration was not limitant (> $80 \%$ saturation). Fish were reared in fibreglass tanks until they reached $0.5 \mathrm{~g}$ and then in concrete raceways until the end of the experiment.

Fish were fed to satiation using extruded commercial feed (Le Gouessant, Lamballe, France): Neo Extra from $40 \mathrm{~g}$ to $300 \mathrm{~g} \mathrm{(43 \%} \mathrm{protein} \mathrm{and} \mathrm{23 \%} \mathrm{lipids)} \mathrm{and} \mathrm{Neo} \mathrm{Ultra} \mathrm{until} \mathrm{the}$ end of the experiment (41\% proteins and $26 \%$ lipids). Density increased progressively to reach $70 \mathrm{~kg} / \mathrm{m}^{3}$. Survival rate was $92.6 \%$ from tagging (d198) to slaughtering (d509).

\subsection{Trait recording}

At the end of the experiment, 2042 fish were randomly sampled and sub-divided into two equal sub-groups, which were then slaughtered at $509 \mathrm{dpf}$ or $511 \mathrm{dpf}$. This allowed limiting the duration of the post-mortem period to less than 5 days. The 2 sub-groups were treated in the same way, under the same conditions: 3 days of fasting, live transportation by truck to the Aqualande processing plant (Roquefort, France, $50 \mathrm{~km}$ from the fish farms) for slaughter $\left(\mathrm{CO}_{2}\right.$ anaesthesia, followed by bleeding in icy water), and then transportation by refrigerated truck to the IFREMER (Nantes, France) for processing and data collection. Fish processing and data collection were done between 511 and $515 \mathrm{dpf}$.

The body length $(\mathrm{BL})$ and body weight $(\mathrm{BW})$ and the following body compartment traits were measured: head weight (HeadW), headless gutted carcass weight (HGCarcW), weight of trimmed non skinned left fillet (LTFW), dorsal (LDTW) and ventral (LVTW) trimming waste weights of the left fillet, and gonad weight (GonadW). These traits were combined to compose synthetic traits (see Table 1): carcass weight (CarcW $=$ HGCarcW + HeadW), fillet weight $\left(\right.$ FilW $=2^{*}($ LTFW + LDTW + LVTW $\left.)\right)$, trimmed waste weight $\left(\right.$ TrimW $=2^{*}($ LDTW + LVTW)), vertebral axis weight $\left(\right.$ AxisW $\left.=\mathrm{HGCarcW}-2^{*} \mathrm{FilW}\right)$, and bone weight $($ BoneW $=$ HeadW + AxisW).

The Fulton coefficient condition was calculated as $\mathrm{K}=\mathrm{BW}(\mathrm{g}){ }^{*} 100 / \mathrm{BL}^{3}(\mathrm{~cm})$. The other traits were divided by the body weight (BW) to calculate the different yields at processing (see Table 1): head yield (Head\%), carcass yield (Carc\%), headless gutted carcass yield 
(HGCarc\%), fillet yield (Fil\%), trimmed fillet yield (TrimFil\%), vertebral axis yield (Axis\%), bone yield (Bone\%), and gonado-somatic index (Gonad\%).

Filleting and trimming were done each by a different person. These persons inter-changed their roles once during the week of processing and this was considered as a potential fixed effect.

Vertebral malformations were evaluated by visual examination of the vertebral axis after filleting of each carcass. Malformed fish $(n=18)$ were excluded from the measurements. Four other fish were discarded due to errors in data collection. The number of fish genotyped was 2020.

\subsection{Genotyping and statistical analysis}

Non malformed fish were assigned to their parents by the French laboratory for livestock genotyping LABOGENA (ISO 17025 accredited, Jouy en Josas, France) using twelve microsatellites organised in 2 panels. Only fish unambiguously assigned to their both parents by exclusion with a maximum of one mismatch allowed were included in the statistical analysis.

Mean and standard deviation for each trait, and fixed effects were estimated using the SAS software package. Non-genetic maternal effect, with dams considered as a random effect ( $\mathrm{n}$ $=60$ ), was not significant for any trait.

Heritability was estimated using VCE 6 software (Groeneveld et al., 2008) with a univariate animal model for each trait:

$Y=X \beta+Z a+\varepsilon$

where $Y$ is the vector of observations, $\beta$ is the vector of fixed effects (overall mean, filetingtrimming operator with 2 levels), $a$ is the vector of random additive genetic effects, $\varepsilon$ is the vector of random residual effects. $X$ and $Z$ are incidence matrices.

Genetic correlations between two traits were estimated with a bivariate animal model, including fixed effects when needed.

Genetic parameters were first estimated using these models on raw data and yields. This provided results that could be used to predict response to selection on body weight at a constant age. However, as yields are ratios, the expected responses to selection on yields may be biased and unpredictable. This is because components of ratios can be highly phenotypically correlated, they can exhibit different variances giving more intensity on the component with greater variation, and finally heritability may results of pure mathematical relationship between genetic parameters of the ratio components (see Pearson (1897) and Sutherland (1965)). As suggested by Koch et al. (1963) to avoid problems listed above, the residual of the linear phenotypic regression between the log-transformed weight of compartments and the log of BW was calculated, a principle routinely applied to estimate e.g. residual feed intake in livestock selection as a surrogate for feed efficency which is a ratio trait (Moore et al., 2008). These new traits were analysed with the above univariate or bivariate animal models to estimate genetic parameters of body compartment developments taking into account their allometric relationship with body weight. 


\section{Results}

Of the 2020 fish genotyped, 2010 were assigned to unique parental pairs, eight to more than 1 couple, and two individuals did not give an appropriate quality of DNA. The rate of correct assignment to one unique couple was then $99.5 \%$.

A total of 559 full-sib families were represented $(93.1 \%$ of the expected 600$)$. Progeny per family varied from 1 to 13 . All 100 sires used at fertilisation were represented in the progeny. The mean number of progeny per sire was $20.1 \pm 7.1$ (S.D.) and varied from 8 to 45 . All 60 dams were also represented. The mean number of progeny per dam was $33.6 \pm 9.6$ (S.D.) and varied from 12 to 61 . Spontaneous triploids $(n=48)$, identified from their DNA-fingerprint signature, were removed from the statistical analysis (see discussion in Haffray et al., 2012). The maximum number of fish used for genetic data treatment for one trait was 1962 and the minimum 1895 (see Tables 2 and 3).

Descriptive statistics (mean, standard deviation, and number of fish used in the analysis) for phenotypic traits are given in Table 2 (weight of body compartments) and in Table 3 (yields). The mean weight at slaughter was $1636 \mathrm{~g}$. The fillet (non-trimmed with skin) represented the major part of the body weight $(66.8 \%)$, while bony tissues accounted for $20.9 \%$. Within the bony tissues, the head and the vertebral axis had the same range of relative quantity $(11.1 \%$ and $9.8 \%$, respectively). The gonads were immature $(0.19 \%$ of BW) and were, therefore, not considered in any further genetic analyses.

No fixed effect was significant for BW, BL, K, CarcW, HeadW, HGCarcW, Carc\%, Head\% or HGCarc\%. A significant "filleting-trimming operator" was observed for FilW, TrimFilW, Fil \%, TrimFil\% and traits derived from the filleting BoneW, AxisW, Axe $\%$ and Bone $\%$.

Heritabilities for BW and BL were intermediate ( 0.37 and 0.30$)$. All other body compartment weights (Table 4$)$ also exhibited medium heritability (0.26 to 0.39$)$ and had high genetic correlations with BW (0.91 to 0.99$)$. K was highly heritable $(0.54)$ and positively genetically correlated with all body compartment weights (0.59 to 0.72 ). All body compartments weights were also highly genetically correlated with one another (0.89 to 0.99$)$.

Heritabilities of yields (Table 5) at the first step of primary processing (Carc\%, HGCarc\%, and $\mathrm{Head} \%)$ were high (0.47 to 0.54$)$. Further processing stages presented intermediate heritabilities ( 0.35 and 0.44 for Fil \%, and TrimFil\%) or, in the case of Bone\%, a somewhat lower (0.22). Only Axis\% presented a low heritability (0.05).

BW was highly genetically correlated $(0.92)$ with body $\mathrm{BL}$, but less so with $\mathrm{K}$. Low genetic correlations ( -0.15 to -0.12$)$ were estimated between $\mathrm{BW}, \mathrm{BL} \mathrm{K}$ with $\mathrm{Carc} \%$. These traits also exhibited low genetic correlations (0.12-0.27) with HGCarc\%,, Fil\% or TrimFil\%. BW and, to a lesser extent, BL were negatively genetically correlated with Head\% (-0.52), Axis\% (-0.48), and Bone\% (-0.57).

Fil\% was highly genetically correlated with Carc\% (0.75), TrimFil\% (0.97) and HGCarc\% (0.97). Fil\% was negatively genetically correlated with $\mathrm{Head} \%(-0.50)$, Axis\% $(-0.31)$ and Bone\% (-0.52).

The linear regressions between the log of the raw traits and the log of BW (Table 6) were all highly significant $(P<0.0001)$ with intermediate to high R-squared values $(0.65$ to 0.99$)$. Head, axis and bones exhibited negative allometry, with regression coefficients lower than 1 ( 0.79 to 0.88 ) indicating that the proportion of body weight comprised of these traits decreased as BW increased phenotypically. 
The heritabilities of the residuals of the linear regression between the log-transformed traits and the log of BW (Table 6) were nearly equal to the heritabilities of yields previously estimated (Table 5). Genetic correlations were also conserved, e.g., between headless gutted carcass and carcass (0.83 and 0.88 ), fillet (0.97 and 0.98$)$, or head ( -0.44 and -0.45 ). The major difference between these traits estimated from the residuals and those estimated on yields was that genetic correlations of the log-transformed residuals of the traits with BW did not significantly differ from zero.

\section{Discussion}

\subsection{Experimental conditions}

This study is the first to report genetic parameters for growth and processing yields in commercial conditions in salmonid families reared together from hatching and a posteriori DNA-pedigreed with microsatellites. Previous experiments using DNA reconstitution of pedigrees have been done with limited experimental designs to estimate family effects (Herbinger et al., 1995, 1999), dam heritability (Fishback et al., 2005) or sire heritability (Chevassus et al., 2002; Dupont-Nivet et al., 2010) or used families pooled after a long period of separate rearing (Powell et al., 2009).

Here, the genetic parameters were estimated using a high number of parents and families. Moreover, the use of a partly factorial design allowed an accurate estimation of genetic parameters and additive effects (Dupont-Nivet et al., 2002). The estimations were obtained in all-female families, which avoided sex effects and/or early male maturation effect (DupontNivet et al., 2010). Moreover, the initial rearing of the families was also set up to limit nongenetic maternal effects (Haffray et al., 2012), which indeed were not significant at slaughter. Moreover, environmental and farming conditions were favourable (see 2.1) and a high survival was observed. The very low rate of vertebral malformation confirms the good rearing conditions and did not allow estimating additive genetic basis for this trait.

\subsection{Heritability for growth and processing traits}

For all the traits measured or calculated, non-genetic maternal effect was not significant. The protocol followed to limit this effect until $60 \mathrm{~g}$ (Haffray et al., 2012) can be estimated efficient to estimate genetic parameters for growth at commercial size or production traits.

Heritabilities of $\mathrm{BW}, \mathrm{BL}, \mathrm{K}$ and $\mathrm{Carc} \%$ are in the upper range of those reported in salmonids (see Gjedrem, 1997 for a review; Kause et al., 2002; Neira et al., 2004; Kause et al., 2007; Powell et al., 2009). This confirms that rainbow trout generally exhibits moderate to high heritabilities for these traits and that the rearing of all families pooled to gather from eyed stage is highly efficient to estimate genetic parameters.

The Fil\% heritability $(0.35)$ is in the upper same range $(0.25-0.38)$ reported in rainbow trout, common carp, European sea bass or Nile tilapia (Kause et al., 2002, 2007 ; Kocour et al., 2007; Saillant et al., 2009; Nguyen et al., 2010). This heritability is much higher than the more limited ones (0.03 to 0.12 ) reported in other publications (Neira et al., 2004; Rutten et al., 2005; Powell et al., 2009; Navarro et al., 2009; Kause et al., 2011; Gjerde et al., 2012) in other species (coho salmon, Nile tilapia, Atlantic salmon, gilthead sea bream or European 
whitefish). A species difference may be a hypothesis to explain these differences. However, very different heritability (from $0.06 \pm 0.04$ to $0.25 \pm 0.07$ ) were estimated within the same species as Nile tilapia (Rutten et al. (2005); Nguyen et al. (2010); Gjerde et al. (2012)) suggesting no general trends and potential effects of reference populations, protocols to estimate genetic parameters and interaction with environmental factors or rearing practices.

Some other specific aspects related to the filleting also have to be considered. Fish were filleted before the initiation of the sexual maturation at the end of spring. Manual filleting was preferred as the literature reported very low heritability with automated filleting (Rutten et al., 2005 and Powell et al., 2009). The filleting of only one fillet (here the left) provided a higher fillet yield than the filleting of the two fillets, and then better estimation of the real biological trait variation. This procedure was validated in a preliminary experiment in trout in witch the fillet yield calculated in doubling the left fillet weight was $1.2 \%$ higher $(61.6 \% \pm 3.4$ vs 60.2 $\% \pm 3.2$ ) than the fillet yield calculated by summing the weight of the two fillets $(n=198$; mean body weight $2.95 \mathrm{~kg} \pm 0.14$; t-test $p<0.05$ ). The lower weight of the second fillet is due to practical difficulties in the filleting of a half carcass. Therefore, this practice may better estimate the real biological differences in muscle yield. Finally, the rearing conditions, with a high oxygen level, may have promoted the expression of the additive genetic component of the muscle development as interaction between oxygen level and muscle development were reported previously (Lefèvre et al., 2007).

The rationale to select on Fil\% has been discussed in tilapia (Rutten et al., 2005; Nguyen et al., 2010; Gjerde et al., 2012) and as BW and FilW are highly genetically correlated, selection on BW has been suggested to be more efficient to improve Fil\%. While Gjerde et al. (2012) did not report improvement of fillet yield in their experiment, the heritability of Fil\% being very low $(0.06 \pm 0.04)$, Bosworth and Wolters (2009) reported positive response to selection on Fil\% or Carc\% in American catfish Ictalurus punctatus. When processing yields exhibit higher heritability than in the cited studies $(0.25$ and 0.06$)$ or when candidates are ranked at constant body weight (our results when raw data are log-transformed), the conclusion may be different and improvement of productivity of the whole production chain different, more specially when considering the financial benefits of processing units. If the improvement of meat yield exhibit limited or lack of responses in experiments reported in mammals (see Nguyen et al., 2010 and Gjerde et al., 2012 for review), selection on breast yield has demonstrated its efficacy in chicken and turkey selection (Le Bihan et al., 2008; Aslam et al., 2011; Case, 2011) as also recently the selection on relative development of tail in fish (Egset et al., 2012). However, the genetic correlation between body weight and breast meat weight was substantially lower (0.76; see Le Bihan-Duval et al., 1999) than those between body weight and the body compartment traits in this paper (0.99). Consequently, the selection response for fillet yield if trout can not be simply expected to be as straightforward as it was reported for breast yield in chicken.

\subsection{Genetic correlation between processing traits}

The high genetic correlations between Fil\% and TrimFil\% (0.96) and the higher heritability of the TrimFil\% (0.44 vs 0.35) can lead this trait to be preferred to Fil\% in selection programs. The lower heritability of Fil\% can indicate environmental variations in the relative dorsal and ventral trimming development or operator variations at filleting. However, we cannot exclude that this lower heritability was also induced by its method of calculation, which involved weighing and summing three different compartment weights.

The very high genetic correlations between the fillet yields (Fil\% or TrimFil\%) and HGCarc\% (0.97) have never been previously reported in fish. Such a high value led us to consider these traits as equivalent. However, the advantage of selection on HGCarc\% is that it could 
avoid filleting during the selection process and thus the costs of labour and raw material depreciation by manipulation. HGCarc\% also has a higher heritability than fillet yield or trimmed fillet yield $(0.54 \mathrm{vs} 0.35)$. Together with the high genetic correlation already reported, selection on this trait should lead to a better response in the target trait (Fil\%) when selecting for the indirect trait (HGCarc\%) than when selecting on the target trait itself. The relative response of $\mathrm{Fil} \%$, comparing indirect selection through $\mathrm{HGCarc \%}$ or direct selection through Fil\%, should be $r=h_{X} r a(X, Y) / h_{Y}=1.49$ (Falconer and MacKay, 1996). To our knowledge, this is one of the rare examples in animal production where selection on an indirect trait should be more efficient than the selection on the trait to be improved, probably because operator bias is avoided (see also Kocour et al., 2007 on common carp). This increase in efficiency is easy to understand. First, $87.4 \%$ of the weight of headless gutted carcass is composed of the fillets. Therefore variation in HGCarc\% mainly represents fillet variation even if some environmental variation from heading and gutting may also exist. Second, when cutting the left fillet of the carcass, there is unavoidable environmental variation due to the different operators i.e. for Fil\% ( $t$-test; $p<0.001$ ) with $67.9 \% \pm 2.0$ for operator $1(n=390)$ vs $66.5 \% \pm 1.9$ for operator $2(n=1531)$. Even with only one operator, there would still be within-operator variation, due to difficulties of precisely following the mediosagittal plane. All within and between operators variations are avoided by using HGCarc\%. The within-operator variation cannot be measured, as one fish cannot be filleted twice. However, the relatively low value of the phenotypic correlation between Fil\% and HGCarc\% ( 0.71 compared with the genetic correlation of 0.97 ) indirectly confirms this hypothesis. Trimming corrects, at least partly, for the mediosagittal error, and we can note that the phenotypic correlation between TrimFil\% and HGCarc\% is slightly higher (0.76) than with untrimmed fillets, although trimming is also subject to operator-associated errors. This result needs to be validated across generations and on other strains or species. Lower genetic correlation could be expected in species with lower fillet yield, as the bones (or the gut) will be the most important body compartment rather than the musculature.

\subsection{Genetic parameters of bony tissues and correlations with production traits}

The sum of the head and the vertebral axis yield (Bone\%) exhibits a lower heritability than head yield $(\mathrm{Head} \%)$. This is linked to the very low heritability estimated for the axis yield (Axis\%) that was estimated by calculation rather than weighing of this tissue. It can be hypothesised that a real anatomical dissection of the vertebral axis could have allowed a better estimation of the genetic variation of the vertebral axis and bone yield.

The genetic bases of the relative development of bony tissues to body weight have received little attention in fish, where only the relative development of head has been investigated. Our values are in the upper range of the heritability estimated for this trait, either as the ratio of head weight to body weight or as the ratio of head length to body weight or length (see Table 7). The three linked bony tissue traits (Head\%, Axis\%, and Bone\%) exhibited high negative genetic correlations with BW, Fil\%, and TrimFil\% yields $(-0.49$ to -0.52$)$. Similar negative correlations were also reported in previous studies in rainbow trout and three other fish species (see Table 7).

However, the correlations between a ratio trait and its denominator can in some cases be largely spurious, without biological meaning (Pearson, 1897). Especially, spurious genetic correlations between a ratio and its denominator can easily occur when the genetic correlation between the numerator and the denominator is close to unity, and the heritabilities and coefficients of variation of the two traits are close to each other (Sutherland, 1965). When we applied Sutherland's formula, which allows estimate the level of the spurious correlation, to our genetic parameters (Table 8), two of the five genetic correlations calculated fit exactly with our estimates of genetic correlation, and the other three were quite 
close, indicating that a significant part of the correlations estimated may be of purely mathematical. Therefore, these genetic correlations need to be considered with high caution as mathematical and biological determinants are not possible to disentangle.

If the genetic correlation estimated with body weight, is of biological origin the present results could demonstrate a potential negative genetic trade-offs between production traits (growth, fillet yield, etc.) and the development of the bony tissues in fish. Based on Falconer and Mackay (1996) formulae for the prediction of correlated genetic gain $\left(\Delta G=i{ }^{*} h_{x}{ }^{*} \sigma_{y}{ }^{*} h_{y}\right.$ * $r_{g}$ ), we estimated a $29.7 \%$ decrease in head yield (from $11.1 \%$ of the total body weight to $7.8 \%$ ) after ten generations of selection for increased body weight with $10 \%$ selection pressure $(i=1.755)$. This high negative correlated response is due to the high heritability of the traits considered (growth, fillet yield and head yield) and their high negative genetic correlations.

The head represents more than $50 \%$ of the bony mass. Head weight and head volume also include the gills, which are the principal organs for most physiological ionic and metabolic homeostasis regulation for respiration, growth, osmoregulation and mineral transfer. As suggested by resource allocation theory (Mignon-Grasteau et al., 2005; Rauw, 2009), lower development of the head (and the vertebral axis) can be associated with a re-orientation of nutrients and/or metabolites (i.e. calcium) from the physiological ossification process to overall growth, especially muscle development, and this re-allocation would be increased if the two traits (growth and fillet yield) are selected together. Preliminary phenotypic investigations in pan-size rainbow trout, in which the role of selection was not estimated, indicated intense bone remodelling by mineral resorption and deposition (Deschamps et al., 2008). Considering the physiological role of the gills in fish and the negative effects on the cardiorespiratory system (located in the head in fish), and as encountered in other livestock species (Rauw et al., 1998), it is crucial to investigate such relationships in fish and to estimate physiological reorientation and potential loss of robustness associated with the potential relative decrease in head size and bony tissues when selecting for production traits. Similarly, it is also important to estimate genetic parameters for the development of other bony tissues, such as fins, which will affect the swimming capacities, scales that affect fish protection against pathogens, and other collagen-rich tissues (as the bones) i.e. Bugeon et al (2010) having observed a higher area of myosepta from trout with higher fillet yield and possible physiological reorientation.

\subsection{Genetic parameters at the same body weight}

When data are log transformed and expressed as residuals to the regression on log(body weight), the trait heritabilities are conserved. Their lack of correlation with body weight illustrated that when allometric relationship is considered, the bigger fish did not have genetically smaller heads, or more fillet. This confirms the lack of correlated response on fillet yield after selection on body weight already reported (Bonnet et al., 2002; Vandeputte et al., 2009b; Gjerde et al., 2012) and the interest to use residuals as demonstrated by Egset et al., (2012) about selection on fish morphology. It can then be expected that fish selected on growth and slaughtered younger, but at constant body weight, should not exhibit a smaller head or lesser bone development. However, fillet yield is still negatively correlated with head weight $(-0.53)$. So, it can be assumed that selection to increase fillet yield would, nevertheless, decrease head yield. These data are preliminary and results need to be confirmed on other data sets and species 


\section{Conclusions}

This work estimated genetic parameters for production traits and non selected bony tissues in rainbow trout. Selection on body weight or fillet yield should lead to a progressive decrease in head and other bony tissues for fish slaughtered at constant age and/or larger sizes. Selection on body weight should not modify the fish morphology when fish are slaughtered at constant body weight, contrary to selection on fillet yield. Whatever the objectives (increased body weight at slaughter or a shortened production cycle at constant body weight), selection on gutted yield should not impair the relative head development. This work highlights the need to carefully monitor these traits through generations of selection and to estimate the impact of head size variation and correlated response on several fitness traits, such as respiratory or adaptive function.

\section{Acknowledgements}

The authors are indebted to the Aqualande staff involved in the production of the animals $(\mathrm{J}$ Y. Gayral, T. Espirat, J. P. Angama, J. F. Lamargot, S. Sétino, V. Petit), the numerous people involved in the data collection: from INRA (G. Choubert, M. Cluzeaud, E. Quillet, J-P. Hiseux, F. Krieg, S. Le Guillou, L. Grima, S. Martineau, M. Perrocheau, L. Lefèvre, S. Launey, J. Leonard, A. Uyanik and F. Vallée), SYSAAF (M. Boulay, P. Rault, and B. Coudurier), IFREMER (I. Baud, B. Bardiot, C., W. Louis, A. Vergnet, and T. Pinazo), and the French breeding companies Viviers de France (E. Willaume, L. Leroux), Font Rome Pisciculture (J-F. Roure) and Les Fils de Charles Murgat (P. Hocdé). We are also grateful to those at Labogena who worked on the DNA parentage assignments (T. Pantano) and those at CIPA for the management of the project (C. Deshayes, M. Levadoux). This work was supported by the French Office of Marine Products OFIMER (convention $n^{\circ} 037 / 05 / \mathrm{C}$ ) and by the EU through IFOP (convention $n^{\circ} 206 / 2005$ ). Authors are thankful to Helen Boudry for the improvement of the English and also to the reviewers for their remarks and proposals of improvement.

\section{References}

Aslam, M., Bastiaansen, J.W.M., Crooijmans, R.P.M.A., Ducro, B.J., Vereijken, A., Groenen, M.A.M., 2011. Genetic variances, heritabilies and maternal effects on body weight, breast meat yield, meat quality traits and the shape of growth curve in turkey birds. BMC Genetics, 12:14.

Bonnet S., Haffray P., Chevassus B., Aubin J., Fauconneau B., 2002. Conformation and carcass quality traits in seawater adult brown trout: correlated responses to selection for freshwater body length growth and triploidy*selection interactions. Aquaculture: 204 (3-4): 193.

Bugeon, J., Lefèvre, F., Cardinal, M., Uyanik, A., Davenel, A., Haffray, P., 2010. Flesh quality in large rainbow trout with high or low fillet yield. J. Muscle Foods 21, 702-721.

Bosworth, B.G.,Wolters, W.R., 2009. Realized heritabilities for meat yield traits in divergently selected lines of channel catfish, Ictalurus punctatus. Aquaculture 247 (1-4), 6.

Case, A.L., 2011. Improving the efficacy of turkey breeding programs through selection index design, technological advancement and management optimization. Thesis, Guelph University, September 2011. 
Chevassus, B., Dupont-Nivet, M., Mauger, S., Haffray, P., Vandeputte, M., 2002. Estimation of heritabilities in two groups of rainbow trout (Oncorhynkus mykiss) with families mixed together since eyed stage. $7^{\text {th }}$ World Congress on Genetics Applied to Livestocks Production. Montpellier, August 19-23, 2002. Book of abstracts, Communication n ${ }^{\circ} 66-$ 09, p 147.

Chevassus B, Quillet E, Krieg F, Hollebecq MG, Mambrini M, Labbe L, Hiseux JP, Vandeputte M, 2004. Enhanced individual selection for selecting fast growing fish: the „PROSPER ${ }^{\text {"wt }}$ method, with application on brown trout (Salmo trutta fario). Gen. Sel. Evol. 36, 643-661.

Deschamps, M.-H., Kacem, A., Ventura, R., Courty, G., Haffray, P., Meunier, F.J., Sire, J.-Y., 2008. Assessment of "discreet" vertebral abnormalities, bone mineralization and bone compactness in farmed rainbow trout. Aquaculture 279, 11-17.

Dupont-Nivet M., Vandeputte M., Chevassus B., 2002. Optimisation of factorial mating designs for inference on heritability in fish species. Aquaculture 204, 361-370.

Dupont-Nivet M., Vandeputte M., Haffray P., Chevassus B., 2006. Effect of different mating designs on inbreeding, genetic variance and response to selection when applying individual selection in fish breeding programs. Aquaculture 252, 161-170.

Dupont-Nivet, M., Chevassus, B., Mauger, S., Haffray, P., Vandeputte, M., 2010. Side effects of sexual maturation on heritability estimates in rainbow trout (Oncorhynchus mykiss). Aquaculture Research 41, e878-e880.

Egset, C. K., Hansen, T. F., Le Rouzic, A., Bolstad, G. H., Rosenqvist, G., Pélabon, C., 2012. Artificial selection on allometry: change in elevation but not slope. J. Evol. Biol., 1-11. doi: 10.1111/j.1420-9101.2012.02487.x.

Falconer, D.S., Mackay, T.F.C., 1996. Introduction to quantitative genetics. Pearson Prentice Hall Harlow, Essex.

Fishback, A.G., Danzmann, R.G., Ferguson, M.M., Gibson, J.P., 2002. Estimates of genetic parameters and genotype by environment interactions for growth traits of the rainbow trout (Oncorhynchus mykiss) as inferred using molecular pedigrees. Aquaculture 206, $137-150$.

Gjedrem, T., 1997. Flesh quality improvement in fish through breeding. Aquacult. Int. 5, 197206.

Gjedrem, T., Thodesen, J., 2005, Selection, In: Gjedrem, T. (Ed.), Selection and Breeding Programs in Aquaculture, Springer, Dordrecht, The Netherlands, pp. 89-111.

Gjerde, B., Mengistu, S.B., Ødegard, J., Johansen, H., Altamirano, D.S., 2012. Quantitative genetics of body weight, fillet weight and fillet yield in Nile tilapia (Oreochromis niloticus). Aquaculture. 342-343, 117-124.

Groeneveld,E., Kovac, M., Mielenz, N., 2008. VCE user's guide and reference manual version 6.0. Friedrich Loeffler Institute, Neustadt, Germany, 125 pp.

Haffray P., Pincent C., Rault P., Coudurier B., 2004. Domestication et amélioration génétique des cheptels piscicoles français dans le cadre du SYSAAF. INRA Prod. Anim. 17(3), 243-252.

Haffray, P., Vandeputte, M., Petit, V., Pincent, C., Chatain, B., Chapuis, H., Mériaux, J.C., Coudurier, B., Quillet, E., Dupont-Nivet, M., 2012. Minimizing maternal effect in salmonids families mixed since eyed stages for selection and a posteriori DNApedigreed. Accepted in Livestock Science.

Havenstein, G.B., Ferket, P.R., Qureshi, M.A., 2003a. Growth, livability, and feed conversion of 1957 versus 2001 broilers when fed representative 1957 and 2001 broiler diets. Poult. Scie. 82, 1500-1508.

Havenstein, G.B., Ferket, P.R., Qureshi, M.A., 2003b. Carcass composition and yield of 1957 versus 2001 broilers when fed "typical" 1957 and 2001 broiler diets. Poult. Sci. 82, 1509-1518.

Herbinger, C. M., Doyle, R. W., Pitman, E. R., Paquet, D., Mesa, K. A., Morris, D. B., Cook, D., 1995. DNA fingerprint analysis of paternal and maternal effects on offspring growth and survival in communally-reared rainbow trout. Aquaculture 137, 245-256. 
Herbinger, C.M., O"Reilly, P.T., Doyle, R.W., Wright, J.M., O'Flynn, F., 1999. Early growth performance of Atlantic salmon full-sib families reared in single family tanks versus in mixed family tanks. Aquaculture 173, 105-116.

Kause, A., Ritola, O., Paananen, T., Mäntysaari, E., Eskelinen, U., 2002. Coupling body weight and its composition: a quantitative genetic analysis in rainbow trout. Aquaculture 211, 65-79.

Kause A., Paananen T., Ritola O., Koskinen H., 2007. Direct and indirect selection of visceral lipid weight, fillet weight, and fillet percentage in a rainbow trout breeding program. J. Anim. Sci. 85, 3218-3227.

Kause, A., Ritola, O., Paananen, T., 2007. Changes in the expression of genetic characteristics across cohorts in steletal deformations of farmed salmonids. Den. Sel. Evol. 39, 529-543.

Kause, A., Quinton, C., Airaksinen, S., Ruohonen, K., Koskela, J., 2011. Quality and production trait genetics of farmed European whitefish, Coregonus lavaretus. J. Anim. Sci. 89, 959-971.

Knap, P.W., Rauw., W.M., 2009. Selection for high production in pigs. In: Rauw, W.M. (Ed.), Resources Allocation Theory Applied to Farm Animal Production. . Cab International, pp. 210-229.

Koch, R. M., L. A. Swiger, D. Chambers, and K. E. Gregory. 1963. Efficiency of feed use in beef cattle. J. Anim. Sci. 22:486-494.

Kocour, M., Mauger, S., Rodina, M., Gela, D., Linhart, O., Vandeputte, M., 2007. Heritability estimates for processing and quality traits in common carp (Cyprinus carpio L.) using a molecular pedigree. Aquaculture, 270, 43-50.

Komen, H., Haffray, P., Kaushik, S., New, M., Olesen, I., Liinamo, A.E., 2002. Defining breeding goals for the future sustainable aquaculture. Aquaculture Europe, December, 11-14.

Le Bihan-Duval, E., S. Mignon-Grasteau, N. Millet, and C. Beaumont, 1998. Genetic analysis of a selection experiment on increased body weight and breast muscle weight as well as on limited abdominal fat weight. Br. Poult. Sci. 39:346-353.

Le Bihan-Duval, E., Début, M., Berri, C.M., Sellier, N., Santé-Loutellier, V., Jego, Y., Beaumont, C., 2008. Chicken meat quality: genetic variability and relationship with growth and muscle characteristics. BMC Genetics, 953.

Lefèvre, F., Aubin, J, Louis, W., Labbé, L., Bugeon, J., 2007. Moderate hypoxia or hyperoxia affect fillet yield and the proportion of red muscle in rainbow trout. Cybium, 31(2), 247253.

Mignon-Grasteau, S., Boissy, A., Bouix, J., Faure, J.M., Fisher, A.D., Hinch, G.N., Jensen, P., Le Neindre, P., Mormède, P., Prunet, P., Vandeputte, M., Beaumont, C., 2005. Genetics of adaptation and domestication in livestock. Livest. Prod. Sci. 93, 3-14.

Moore, S.S., Mujibi, F.D., Sherman, E.L., 2008. Molecular basis for residual feed intake in beef cattle. J. Anim. Sci.,87, 41-47.

Navarro, A., Zamorano, M.J., Hildebrandt, S., Ginés, R., Aguilera, C., Afonso, J.M., 2009. Estimates of heritabilities and genetic correlations for growth and carcass traits in gilthead seabream (Sparus auratus L.), under industrial conditions. Aquaculture 289, 25-230.

Neira, R., Lhorente, J.P., Aranedaı, C., Dıaz, N., Bustos, E., Alert, A., 2004. Studies on carcass quality traits in two populations of Coho salmon (Oncorhynchus kisutch): phenotypic and genetic parameters. Aquaculture 241, 117-131.

Nguyen, H.H., Ponzoni, R.W., Abu-Bakar, K.R., Hamzah, A., Khaw, H.L., Yee, H.Y., 2010. Correlated response in fillet weight and yield to selection for increased harvest weight in genetically improved farmed tilapia (GIFT strain), Oreochromis niloticus. Aquaculture $305,1-5$.

Pearson, K., 1897. Mathematical contributions to the theory of evolution. On a form of spurious correlation which may arise when indices are used in the measurement of organ. Proc. Roy. Soc. (London) 60, 489-498. 
Powell, J., White, I., Guy, D., Brotherstone, S., 2009. Genetic parameters of production traits in Atlantic salmon (Salmo salar). Aquaculture 274, 225-231.

Rauw, W.M., Kanis, E., Noordhuizen-Stassen, E.N., Grommers, F.J., 1998. Undesirable side effects of selection for high performance efficiency in farm animals: a review. Livest. Prod. Sci. 56, 15-33.

Rauw, W.M. (Ed.), 2009. Resources Allocation Theory Applied to Farm Animal Production. Cab International, $344 \mathrm{p}$.

Rutten J.M., Bovenhuis H., Komen H., 2005, Genetic parameters for fillet traits and body measurements in Nile tilapia (Oreochromis niloticus L.). Aquaculture 246, 125-132.

Saillant, E.., Dupont-Nivet, M., Sabourault, M., Haffray, P., Laureau S., Vidal, M-O., Chatain, B., 2009. Genetic variation for carcass quality traits in cultured sea bass (Dicentrarchus labrax). Aquat. Living Resourc. 22, 105-112.

Sanchez, A., Plouzeau, M., Rault, P., Picard, M., 2000. Croissance musculaire et fonction cardio-respiratoire chez le poulet de chair. INRA Prod. Anim., 13, 37-45.

Siegel, P.B., Honaker, C.F., Rauw, W.M., 2009. Selection for high production in poultry. In: Rauw, W.M. (Ed.), Resources Allocation Theory Applied to Farm Animal Production. Cab International, pp. 230-242.

Sutherland, T.M., 1965. The correlation between feed efficiency and rate of gain, a ratio and its denominator. Biometrics 21, 739-749.

Vandeputte M., Dupont-Nivet, M., Haffray, P., Chavanne H., Cenadelli, S., Parati, K., Vidal M.O., Vergnet A., Chatain, B., 2009a. Response to domestication and selection for growth in the European sea bass (Dicentrarchus labrax) in separate and mixed tanks. Aquaculture 286, 20-27.

Vandeputte, M., Bugeon, J., Dupont-Nivet, M., Chavanne, H., Haffray, P., Lefèvre, F., Labbé, L., Vergnet, A., Chatain, B., 2009b. Processing quality of selected fish: quasi-absence of correlated responses to selection on growth in the brown trout Salmo trutta and the sea bass Dicentrarchus labrax. EAS Aquaculture Europe Conference, August 14-17, 2009, 627-628.

van der Most, P.J., de Jong, B., Parmentier, H.K., Verhulst, S., 2011. Trade-off between growth and immune function: a meta-analysis of selection experiments. Func. Ecol. 25, 74-80. 


\section{Tables}

Table 1: Definition of the different synthetic weight and ratio traits calculated in rainbow trout Oncorhynchus mykiss, and raw traits measured at the processing plant. The primary traits used to calculate these new synthetic traits were: body length (BL) whole body weight (BW), head weight (HeadW), gonad weight (GonadW), headless gutted carcass weight (GHCarcW), left trimmed fillet weight (LTFW), and dorsal (LDTW) and ventral (LVTW) trimming waste weights of the left fillet.

\begin{tabular}{|c|c|c|}
\hline Compartment name & Weight traits & Yield traits \\
\hline Head & HeadW & Head $\%=$ HeadW * $100 /$ BW \\
\hline Gutted headless carcass & HGCarcW & HGCarc\% $=$ HGCarcW * $100 /$ BW \\
\hline Condition coefficient & - & $\mathrm{K}=\mathrm{BW}^{3} * 100 / \mathrm{BL}$ \\
\hline Carcass & CarcW= HGCarcW + HeadW & Carc\% $=$ CarcW * $100 /$ BW \\
\hline Trimming waste & TrimW $=2^{*}($ LDTW + LVTW $)$ & Trim $\%=$ TrimW * $100 /$ BW \\
\hline Fillet & FilW $=2{ }^{*}($ LDTW + LVTW + LTFW $)$ & Fil\% $\%$ FilW * $100 /$ BW \\
\hline Trimmed fillet & TrimFilW $=2{ }^{*}$ LTFW & TrimFil\% $=$ TrimFilW * $100 /$ BW \\
\hline Vertebral axis & AxisW = HGCarcW - FiletW & Axis $\%=A x i s W * 100 / B W$ \\
\hline Bone & BoneW = HeadW + AxisW & Bone $\%=$ BoneW * $100 /$ BW \\
\hline Gonad & GonadW & Gonad $\%=$ GonadW * $100 /$ BW \\
\hline
\end{tabular}


Table 2: Mean \pm standard deviation, maximum, minimum and number of fish measured $(\mathrm{N})$ in rainbow trout Oncorhynchus mykiss for body weight (BW), body length (BL), condition coefficient $(\mathrm{K})$, and the weight for the following traits: carcass (CarcW), head (HeadW), headless gutted carcass (HGCarcW), trimming waste from both fillets (TrimW), both trimmed fillets (TrimFilW), both fillets (FilW), vertebral axis (AxisW), head and vertebral axis (BoneW).

\begin{tabular}{|l|c|c|c|c|}
\hline Trait & Mean \pm SD & Minimum & Maximum & $\mathrm{N}=$ \\
\hline BW $(\mathrm{g})$ & $1639.2 \pm 350.9$ & 424.5 & 2801.5 & 1962 \\
\hline $\mathrm{BL}(\mathrm{cm})$ & $48.1 \pm 3.0$ & 36.2 & 55.2 & 1958 \\
\hline $\mathrm{K}\left(100^{*} \mathrm{~g} / \mathrm{cm}^{3}\right)$ & $1.46 \pm 0.11$ & 1.08 & 1.93 & 1935 \\
\hline CarcW $(\mathrm{g})$ & $1436.1 \pm 306.3$ & 386.0 & 2499.9 & 1951 \\
\hline HeadW $(\mathrm{g})$ & $180.5 \pm 32.4$ & 59.5 & 276.2 & 1929 \\
\hline HGCarcW $(\mathrm{g})$ & $1256.0 \pm 270.2$ & 326.5 & 2224.0 & 1916 \\
\hline TrimW $(\mathrm{g})$ & $86.4 \pm 22.7$ & 17.0 & 182.8 & 1929 \\
\hline TrimFilW (g) & $1009.9 \pm 222.1$ & 249.8 & 1794.8 & 1929 \\
\hline FilW (g) & $1096.3 \pm 240.8$ & 273.0 & 1977.6 & 1929 \\
\hline AxisW (g) & $159.3 \pm 38.7$ & 52.8 & 328.5 & 1916 \\
\hline BoneW (g) & $339.8 \pm 66.7$ & 113.0 & 600.3 & 1916 \\
\hline Gonads (GonadW) & $3.16 \pm 1.51$ & 0.0 & 19.6 & 1895 \\
\hline
\end{tabular}

Table 3: Mean yield ( \pm standard deviation) maximum, minimum and number of fish $(\mathrm{N})$ in rainbow trout Oncorhynchus mykiss for carcass yield (Carc\%), head yield (Head\%), headless gutted carcass yield (HGCarac\%), fillet yield (Fil\%), trimmed fillet yield (TrimFil\%), trimming yield (Trim\%), vertebral axis yield (Axis\%), bone yield (Bone\%), gonad yield (Gonad\%).

\begin{tabular}{|l|c|c|c|c|}
\hline Trait & Mean \pm SD & Minimum & Maximum & Effective \\
\hline Carc\% & $87.7 \pm 1.4$ & 82.3 & 93.4 & 1948 \\
\hline Head\% & $11.1 \pm 0.9$ & 8.3 & 14.9 & 1925 \\
\hline HGCarac\% & $76.6 \pm 1.5$ & 71.0 & 81.4 & 1913 \\
\hline Fil\% & $66.8 \pm 2.0$ & 59.7 & 73.6 & 1925 \\
\hline TrimFil\% & $61.5 \pm 1.9$ & 55.0 & 67.4 & 1925 \\
\hline Axis\% & $9.8 \pm 1.4$ & 4.4 & 15.2 & 1913 \\
\hline Bone\% & $20.9 \pm 1.8$ & 14.8 & 27.2 & 1913 \\
\hline Gonad\% & $0.19 \pm 0.07$ & 0.00 & 0.81 & 1895 \\
\hline
\end{tabular}


Table 4: Heritability estimates ( \pm standard error) (diagonal), phenotypic correlations (below the diagonal) and genetic correlations \pm standard error (above the diagonal) in rainbow trout Oncorhynchus mykiss for body weight (BW), body length (BL), condition coefficient (K), and the following weight traits: carcass (CarcW), head (HeadW), headless gutted carcass (HGCarcW), trimming waste from both fillets (TrimW), both trimmed fillets (TrimFilW), both fillets (FilW), vertebral axis (AxisW), head and vertebral axis (BoneW).

\begin{tabular}{|l|l|l|l|l|l|l|l|l|l|l|}
\hline & BW & BL & K & CarcW & HeadW & HGCarcW & TrimFilW & FilW & BoneW & AxisW \\
\hline BW & $\mathbf{0 . 3 7} \mathbf{0 . 0 4}$ & $0.92 \pm 0.02$ & $0.70 \pm 0.04$ & $0.99 \pm 0.01$ & $0.91 \pm 0.01$ & $0.99 \pm 0.01$ & $0.99 \pm 0.01$ & $0.99 \pm 0.01$ & $0.98 \pm 0.01$ & $0.96 \pm 0.01$ \\
\hline BL & 0.93 & $\mathbf{0 . 3 0} \pm \mathbf{0 . 0 4}$ & $0.35 \pm 0.07$ & $0.92 \pm 0.02$ & $0.86 \pm 0.02$ & $0.91 \pm 0.02$ & $0.90 \pm 0.02$ & $0.91 \pm 0.01$ & $0.92 \pm 0.01$ & $0.94 \pm 0.02$ \\
\hline K & 0.59 & 0.27 & $\mathbf{0 . 5 4} \pm \mathbf{0 . 0 4}$ & $0.70 \pm 0.04$ & $0.59 \pm 0.05$ & $0.71 \pm 0.04$ & $0.72 \pm 0.04$ & $0.72 \pm 0.04$ & $0.61 \pm 0.05$ & $0.60 \pm 0.06$ \\
\hline CarcW & 0.99 & 0.93 & 0.58 & $\mathbf{0 . 3 7} \pm \mathbf{0 . 0 4}$ & $0.91 \pm 0.01$ & $0.99 \pm 0.01$ & $0.99 \pm 0.01$ & $0.99 \pm 0.01$ & $0.97 \pm 0.01$ & $0.99 \pm 0.01$ \\
\hline HeadW & 0.93 & 0.89 & 0.47 & 0.93 & $\mathbf{0 . 3 9} \pm \mathbf{0 . 0 4}$ & $0.88 \pm 0.01$ & $0.86 \pm 0.02$ & $0.87 \pm 0.02$ & $0.92 \pm 0.02$ & $0.98 \pm 0.01$ \\
\hline HGCarcW & 0.99 & 0.92 & 0.57 & 0.99 & -0.63 & $\mathbf{0 . 3 6} \pm \mathbf{0 . 0 4}$ & $0.99 \pm 0.01$ & $0.99 \pm 0.01$ & $0.97 \pm 0.01$ & $0.95 \pm 0.01$ \\
\hline TrimFilW & 0.99 & 0.92 & 0.58 & 0.99 & -0.63 & 0.99 & $\mathbf{0 . 3 6} \pm \mathbf{0 . 0 4}$ & $0.99 \pm 0.01$ & $0.93 \pm 0.01$ & $0.97 \pm 0.01$ \\
\hline FilW & 0.99 & 0.92 & 0.58 & 0.99 & -0.63 & 0.99 & 0.99 & $\mathbf{0 . 3 5} \pm \mathbf{0 . 0 4}$ & $0.94 \pm 0.01$ & $0.97 \pm 0.01$ \\
\hline BoneW & 0.91 & 0.87 & 0.46 & 0.92 & -0.40 & 0.91 & 0.87 & 0.86 & $\mathbf{0 . 3 4} \pm \mathbf{0 . 0 4}$ & $0.98 \pm 0.01$ \\
\hline AxisW & 0.79 & 0.75 & 0.40 & 0.79 & -0.44 & 0.79 & 0.74 & 0.73 & 0.95 & $\mathbf{0 . 2 6} \pm \mathbf{0 . 0 3}$ \\
\hline
\end{tabular}


Table 5: Heritability ( \pm standard error) estimates (diagonal), phenotypic correlations (below the diagonal) and genetic correlations \pm standard error (above the diagonal) in rainbow trout Oncorhynchus mykiss for body weight (BW), body length (BL), condition coefficient (K), carcass yield $($ Carc\%), head yield (Head\%), headless gutted carcass yield (HGCarc\%), trimmed fillet yield (TrimFil\%), fillet yield (Fil\%), bone yield (Bone\%), vertebral axis yield (Axis\%).

\begin{tabular}{|c|c|c|c|c|c|c|c|c|c|c|}
\hline & BW & $\mathrm{BL}$ & $\mathrm{K}$ & Carc\% & Head $\%$ & HGCarc\% & TrimFil\% & Fil\% & Bone $\%$ & Axis $\%$ \\
\hline BW & $0.37 \pm 0.04$ & $0.92 \pm 0.02$ & $0.70 \pm 0.04$ & $-0.15 \pm 0.07$ & $-0.52 \pm 0.05$ & $0.14 \pm 0.08$ & $0.21 \pm 0.07$ & $0.22 \pm 0.08$ & $-0.57 \pm 0.06$ & $-0.48 \pm 0.14$ \\
\hline $\mathrm{BL}$ & 0.93 & $0.30 \pm 0.04$ & $0.35 \pm 0.07$ & $-0.12 \pm 0.07$ & $-0.43 \pm 0.06$ & $0.12 \pm 0.08$ & $0.15 \pm 0.08$ & $0.15 \pm 0.09$ & $-0.43 \pm 0.07$ & $-0.27 \pm 0.15$ \\
\hline $\mathrm{K}$ & 0.59 & 0.27 & $0.54 \pm 0.04$ & $-0.14 \pm 0.07$ & $-0.46 \pm 0.06$ & $0.12 \pm 0.07$ & $0.26 \pm 0.07$ & $0.27 \pm 0.08$ & $-0.67 \pm 0.11$ & $-0.60 \pm 0.06$ \\
\hline Carc\% & -0.18 & -0.10 & -0.25 & $0.49 \pm 0.04$ & $0.13 \pm 0.07$ & $0.83 \pm 0.02$ & $0.74 \pm 0.03$ & $0.75 \pm 0.03$ & $0.18 \pm 0.15$ & $0.20 \pm 0.15$ \\
\hline Head\% & -0.61 & -0.51 & -0.48 & 0.25 & $0.47 \pm 0.03$ & $-0.44 \pm 0.06$ & $-0.50 \pm 0.06$ & $-0.50 \pm 0.06$ & $0.95 \pm 0.02$ & $0.44 \pm 0.01$ \\
\hline HGCarc\% & 0.20 & 0.21 & 0.04 & 0.81 & -0.37 & $0.54 \pm 0.04$ & $0.96 \pm 0.01$ & $0.97 \pm 0.01$ & $-0.36 \pm 0.08$ & $-0.06 \pm 0.14$ \\
\hline TrimFil\% & 0.26 & 0.24 & 0.15 & 0.54 & -0.40 & 0.76 & $0.44 \pm 0.04$ & $0.97 \pm 0.01$ & $-0.49 \pm 0.06$ & $-0.24 \pm 0.14$ \\
\hline Fil\% & 0.26 & 0.24 & 0.15 & 0.48 & -0.40 & 0.71 & 0.92 & $0.35 \pm 0.04$ & $-0.52 \pm 0.06$ & $-0.31 \pm 0.14$ \\
\hline Bone\% & -0.44 & -0.35 & -0.37 & 0.24 & 0.64 & -0.16 & -0.60 & -0.73 & $0.22 \pm 0.03$ & $0.70 \pm 0.08$ \\
\hline Axis \% & -0.17 & -0.12 & -0.18 & 0.15 & 0.18 & 0.03 & -0.52 & -0.68 & 0.87 & $0.05 \pm 0.02$ \\
\hline
\end{tabular}


Table 6: Heritability ( \pm standard error) estimates (diagonal) and genetic correlations \pm standard error (above the diagonal) in rainbow trout Oncorhynchus mykiss estimated for the residuals $(\mathrm{R})$ of the linear regression between the log of body weight (BW) and the other body compartments: carcass weight (CarcW), headless gutted carcass weight (HGCarcW), head weight (HeadW), fillet weight (FilW), trimmed fillet weight (TrimFilW), vertebral axis weight (AxisW) and bone weight (BoneW). a = Regression coefficient of the linear regression between the log of the trait and the log of body weight $(\mathrm{BW}), \mathrm{R}^{2}=$ determination coefficient of the regression.

\begin{tabular}{|c|c|c|c|c|c|c|c|c|c|}
\hline & BW & $\mathrm{R}(\mathrm{BW})$ & $\mathrm{R}($ CarcW $)$ & $\mathrm{R}(\mathrm{HGCarcW})$ & $\mathrm{R}($ HeadW) & R(FilW) & R(FilTrimW) & $\mathrm{R}(\mathrm{AxeW})$ & $\mathrm{R}($ BoneW) \\
\hline BW & $0.36 \pm 0.06$ & $0.99 \pm 0.001$ & $-0.02 \pm 0.09$ & $-0.03 \pm 0.11$ & $0.01 \pm 0.11$ & $-0.11 \pm 0.12$ & $-0.05 \pm 0.11$ & $0.20 \pm 0.26$ & $0.11 \pm 0.14$ \\
\hline $\mathrm{R}(\mathrm{BW})$ & & $0.32 \pm 0.05$ & $-0.06 \pm 0.19$ & $-0.05 \pm 0.11$ & $-0.01 \pm 0.11$ & $-0.12 \pm 0.13$ & $-0.06 \pm 0.05$ & $0.16 \pm 0.26$ & $0.07 \pm 0.04$ \\
\hline $\mathrm{R}($ CarcW) & & & $0.49 \pm 0.06$ & $0.88 \pm 0.03$ & $0.03 \pm 0.11$ & $0.79 \pm 0.05$ & $0.79 \pm 0.05$ & $0.45 \pm 0.26$ & $0.19 \pm 0.11$ \\
\hline $\mathrm{R}(\mathrm{HGW})$ & & & & $0.55 \pm 0.06$ & $-0.45 \pm 0.06$ & $0.98 \pm 0.02$ & $0.96 \pm 0.02$ & $0.23 \pm 0.27$ & $-0.30 \pm 0.13$ \\
\hline $\mathrm{R}($ HeadW) & & & & & $0.56 \pm 0.06$ & $-0.53 \pm 0.09$ & $-0.52 \pm 0.08$ & $0.33 \pm 0.25$ & $0.94 \pm 0.05$ \\
\hline $\mathrm{R}(\mathrm{FilW})$ & & & & & & $0.31 \pm 0.05$ & $0.97 \pm 0.001$ & $0.03 \pm 0.35$ & $-0.45 \pm 0.11$ \\
\hline $\mathrm{R}$ (FilTrimW) & & & & & & & $0.44 \pm 0.06$ & $0.10 \pm 0.32$ & $-0.42 \pm 0.11$ \\
\hline $\mathrm{R}(\mathrm{AxeW})$ & & & & & & & & $0.02 \pm 0.02$ & $0.62 \pm 0.16$ \\
\hline $\mathrm{R}$ (BoneW) & & & & & & & & & $0.17 \pm 0.04$ \\
\hline$a$ & & & 0.986 & 1.016 & 0.786 & 1.036 & 1.034 & 0.881 & 0.831 \\
\hline $\mathrm{R}^{2}$ & & & 0.995 & 0.993 & 0.891 & 0.985 & 0.985 & 0.648 & 0.856 \\
\hline
\end{tabular}


Table 7: Heritability $\left(\mathrm{h}^{2}\right)$ of relative head development in fish species and genetic correlations ( $\mathrm{rg}$ ) with body weight (BW) and fillet yield (Fil\%).

\begin{tabular}{|l|c|c|l|l|l|}
\hline \multicolumn{1}{|c|}{ Traits } & \multicolumn{1}{|c|}{$\mathrm{h}^{2}$} & $\mathrm{rg} / \mathrm{BW}$ & \multicolumn{1}{|c|}{ Species $/$ Fil\% } & \multicolumn{1}{|c|}{ Authors } \\
\hline Head weight / BW & $0.23 \pm 0.04$ & $-0.78 \pm 0.23$ & $-0.94 \pm 0.21$ & Oreochromis niloticus & Rutten et al., 2005 \\
\hline Head volume / BW & $0.12 \pm 0.08$ & $-0.17 \pm 0.11$ & $-0.17 \pm 0.11$ & Oncorhynchus mykiss & Kause et al., 2007 \\
\hline Head length / Body length & $0.54 \pm 0.12$ & $-0.26 \pm 0.12$ & $-0.23 \pm 0.05$ & Cyprinus carpio & Kocour et al., 2007 \\
\hline Head weight / BW & $0.87 \pm 0.23$ & $-0.83 \pm 0.09$ & $-0.73 \pm 0.15$ & Dicentrachus labrax & Saillant et al., 2009 \\
\hline Head weight / BW & $0.47 \pm 0.03$ & $-0.52 \pm 0.05$ & $-0.50 \pm 0.06$ & Oncorhynchus mykiss & present study \\
\hline
\end{tabular}

Table 8: Genetic correlations estimated (this work) or calculated (according to Sutherland, 1965) between body weight (trait 1) and ratio with between another trait (trait 2) and body weight (trait 1) at their denominator. $\mathrm{h}_{1}{ }^{2}$ and $\mathrm{h}_{2}{ }^{2}$ are heritability of trait 1 and trait 2; $C_{\mathrm{P} 1}$ and $\mathrm{C}_{\mathrm{P} 2}$ are trait 1 and trait 2 coefficient of variation; $r_{\mathrm{G} 12}=$ genetic correlation estimated between trait 1 and trait 2 .

\begin{tabular}{|c|c|c|c|c|c|c|c|}
\hline Trait 2 & $\mathrm{~h}_{1}{ }^{2}$ & $\mathrm{~h}_{2}{ }^{2}$ & $r_{\mathrm{G} 12}$ & $\mathrm{C}_{\mathrm{P} 1}$ & $\mathrm{C}_{\mathrm{P} 2}$ & $\begin{array}{l}\text { Observed rg } \\
\text { between } \\
\text { ratio and BW } \\
\text { (this work) }\end{array}$ & $\begin{array}{l}\text { Calculated } r_{g} \\
\text { according to } \\
\text { Sutherland }\end{array}$ \\
\hline CarcW & 0.37 & 0.37 & 0.99 & 0.2135 & 0.2131 & -0.15 & -0.09 \\
\hline FillW & 0.37 & 0.35 & 0.99 & 0.2135 & 0.2190 & 0.22 & -0.09 \\
\hline HeadW & 0.37 & 0.39 & 0.91 & 0.2135 & 0.1795 & -0.52 & -0.51 \\
\hline AxisW & 0.37 & 0.26 & 0.96 & 0.2135 & 0.2429 & -0.48 & -0.30 \\
\hline BoneW & 0.37 & 0.34 & 0.98 & 0.2135 & 0.1963 & -0.61 & -0.61 \\
\hline
\end{tabular}

${ }^{*} r_{g}=r_{G 12} h_{2} C_{p 2}-h_{1} C_{P 1} /\left(\sqrt{ }\left(h_{2}^{2} C_{P 2}^{2}+h_{1}^{2} C_{P 1}^{2}-2 r_{G 12} h_{1} C_{P 1} h_{2} C_{P 2}\right)\right.$ 